\title{
INCREASING THE SAFETY OF AGRICULTURAL MACHINERY OPERATING ON SLOPING GROUNDS BY PERFORMING STATIC AND DYNAMIC TESTS OF STABILITY ON A NEW-CONCEPT FACILITY
}

\author{
MARCO BIETRESATO \& FABRIZIO MAZZETTO \\ Free University of Bozen-Bolzano, Faculty of Science and Technology - Fa.S.T., Italy.
}

\begin{abstract}
Using agricultural machines on slopes is very risky for operators: drivers difficultly have a correct perception of the stability condition of their vehicles when travelling, especially because it is impossible to instantly check the ground elevation and harshness in correspondence of each wheel. Moreover, the tests that are usually performed to characterize these machines' stability are scarcely helpful in real conditions: these tests check the lateral overturning (maximum angle) in two static configurations only. Evidently, these do not cover most of real situations, do not locate its centre-of-mass and do not consider the centrifugal force (causing load-transfers and dynamically-variable readjustments of the machine's trim in turnings), thus making impossible the setup of eventual active/passive safety-systems to be installed on vehicles.

Therefore, with the aim of overcoming the limitations of actual tests, giving a higher level of information to the tractors' manufacturers and users, we have conceived a completely-new test-apparatus to be installed within our laboratory: the Tiltable Platform. It integrates two subsystems, illustrated hereinafter.

(1) The tiltable/angleable plane is a wide flat structure $(15 \times 15 \mathrm{~m})$, able to simulate different groundgradients, allowing a vehicle to manoeuvre/travel on it along circular paths in a controlled and safe environment. It can also generate an angle at half of its width, transversal to the maximum-slope direction (simulating the exits of an agricultural machine from the inter-rows).

(2) The tilting turntable: has a circular shape and is divided into quadrants, each capable of measuring the weight sustained due to a motionless vehicle positioned on them; it is installed on a tilting structure and can rotate around a vertical axis, thus allowing to vary the angular position of the vehicle's longitudinal axis with reference to the maximum-slope direction.
\end{abstract}

Finally, this test-rig will be useful to develop new test-methodologies to certify the agricultural machines' stability in real operating-conditions (e.g. operating with ballast/mounted-implements).

Keywords: stability of agricultural machines on slopes, static tests of stability, dynamic tests of stability, innovative test-equipment, tiltable/angleable plane, tilting turntable.

\section{INTRODUCTION}

Agricultural mechanization is the process of using machines as power sources to execute many different works of agriculture, with the aim of increasing farm workers' productivity and, at the end, field yields too. In the last decades, powered machinery has progressively replaced manual labour or working animals in many farm jobs, even performed in environments presenting serious difficulties for a machine to operate, e.g. with: difficult accesses to the fields, narrow spaces for manoeuvring and between the inter-rows, loose or not-compact grounds, soils with a pronounced surface roughness, sloping lands. The difficulties for an agricultural machine in the described situations are mainly due to its absolute size and total mass, but also to the propulsion systems adopted to make them travel on the ground (wheels, tracks), intrinsically less flexible than legs. Moreover, with the exception of very rare cases, there is a substantial impossibility for a machine to redistribute its masses and change 
dynamically its trim according to the needs, differently from what any living being (man or animal) would do when it has to climb on slopes or pass through very narrow passages. For the explained reasons, operating with machines (and, in particular, with tractors) could be, in some cases, difficult or, even, dangerous for operators, due to the serious accidents that can happen. To limit the criticalities, it appear evident that at least the environmental and safety issues must be considered together when developing a new agricultural machine, starting even from the phase of the concept and design definition. Any new solution in the field of agricultural mechanization must therefore be proposed taking into account a multiplicity of factors, hence with a vision that is: multifocal (different aspects of the same system to be considered), multidisciplinary (different types of involved subjects/required knowledge), theoretical and experimental at the same time. According to this approach, there are four elements that need to be always considered together when proposing an innovation (here: an agricultural machine or a subsystem for it), and which, altogether, constitute the agricultural mechanization in its more general definition (Fig. 1): (1) the technology, adopted or under development, (2) the labour, to be intended as all the aspects related to human workers, (3) the environment, in which the machine operates, (4) the company/enterprise, i.e. the interfacing with the other organizational elements.

Although the environmental requirements are often the main driver in the development of new technical solutions (by evidencing new needs to pursue and imposing constraints to comply with), the safety and the quality of operators' daily work must always be a primary objective because they are a very important evaluation element of the goodness of a machine's design. Indeed, within the category 'labour' there are lots of very important aspects that cannot be absolutely disregarded nowadays: the worker's safety, ergonomics and comfort, the accidents' prevention, the design, control and rational management of the work place, and the workers' professional education and training.

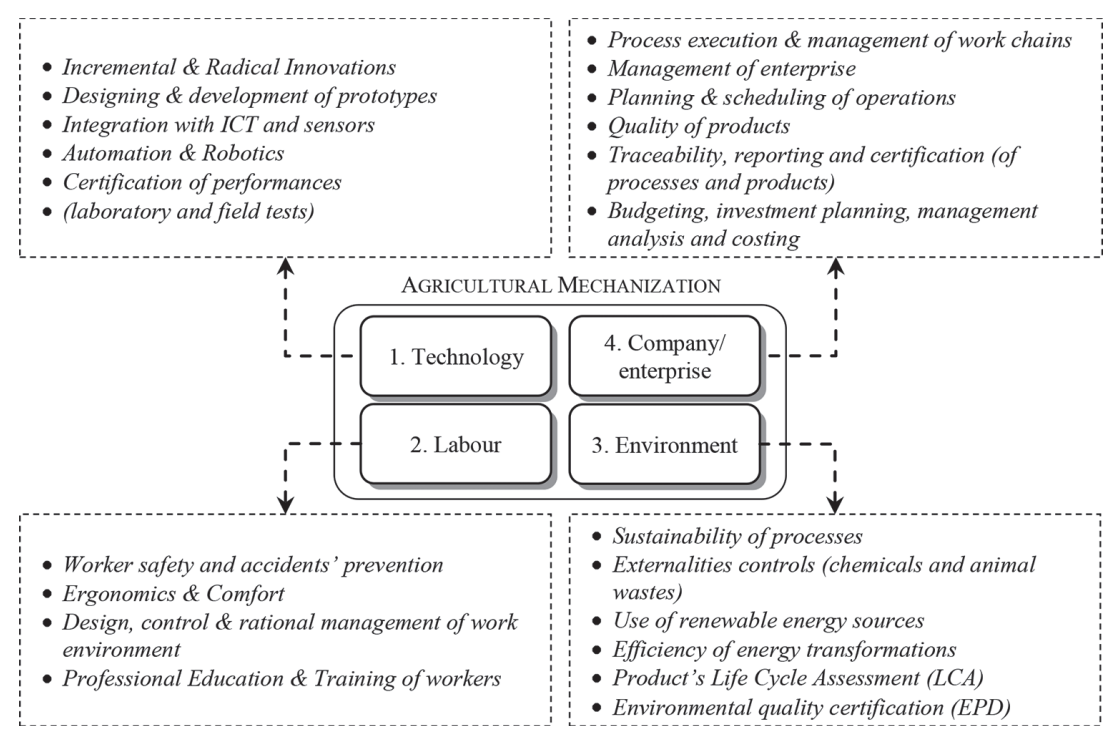

Figure 1: Different aspects of agricultural mechanization [1]; as visible from the scheme, safety issues (related to 'labour' aspects) have the same importance of other, more traditional, issues (e.g., related to the technology). 
Finally, whichever the considered application-sector, there is a need to ensure lasting performances of these technologies (existing or completely new) and, from the point of view of the evaluation, to certify periodically the machine's performances through objective and repeatable measures.

From all the above-explained considerations, it follows that the development of an innovative test-facility inquiring the safety issues of agricultural machines is absolutely a priority for the agricultural-mechanical sector. Indeed, such a facility will be useful to: (1) understand better the intrinsic limits of new prototypes or existing machines in many operating configurations; (2) set up the stability tests in a controlled and safe environment with the aim to define new technical solutions or refine existing solutions for enhancing the safety of agricultural machines; (3) certify the performances of the machines in standardized tests, in order to let the customers make impartial comparisons and trigger a healthy competition among the manufacturers, leading to an improvement of their products.

The present work is organized as follows: after considering the stability issues and the causes of rollover of agricultural machines, actual tests are analysed critically, evidencing their limits, then the development process of new stability tests and the definition of a test facility capable to perform them is reported; after that, the innovative test facility and its main systems are presented in detail.

\section{STABILITY ISSUES AND CAUSES OF ROLLOVER FOR AGRICULTURAL MACHINES}

Among all safety issues suffered by people working in the agricultural sector, the main cause of fatalities is an incorrect/improper use of tractor or of some tractor's subsystems [2]: machinery-related incidents were 32\% of the fatal injuries in the U.S. in 1998 [3]. In particular, one of the most critical issue for farm tractors' drivers is related to the loss of stability of agricultural machines (and, in particular, of machines operating in sloping grounds) leading to the rollover of the machine [4-7]. This condition happens when the projection of the centre of gravity (COG) on the support plane falls outside the vehicle's support polygon. The direction of projection is parallel to the direction of the resultant force (applied to the vehicle's $\mathrm{COG}$ ). A sideways overturning or a lateral rollover of an agricultural machine is not a so-rare event as it can be triggered by the existence of one (or more) of these three, quite common, conditions [8-11]:

1. Excessive ground-gradient; a machine is travelling on a support plane that is globally flat but not horizontal, in particular, the most general situation is when the machine's travelling direction is different from the maximum slope direction of the ground;

2. Critical surface harshness; a machine is travelling on a support plane that is horizontal but not uniformly flat, in particular the machine meets a local modification of the ground slope (e.g. an obstacle, a rut or a hole in the trajectory of a wheel) and one wheel can lose the contact with the soil, thus modifying the support polygon [12];

3. Excessive turning speed; the driver attempts to execute a manoeuvre at a speed incompatible with the desired turning radius, in particular the so-produced centrifugal force deviates the weight force from the direction perpendicular to the support plane till the resultant's projection falls outside the support polygon.

The three described critical conditions can also be divided into two groups, depending on the higher or lower influence/importance of the vehicle's dynamics on the occurrence of these. 
Specifically, the first two conditions can be defined as 'static conditions' (or better, 'quasistatic conditions', assuming that a vehicle has somehow moved from previous safe conditions before reaching one of these critical conditions), the latter one is instead a 'dynamic condition'. By taking into account the many differences and analogies between them (hence, the above-made subdivision in conceptually-homogeneous groups), it is possible to develop some ad-hoc tests that allow investigating the performance of a machine in relation to the many variables that can intervene in determining one of the situations above, for example: the angle between the vehicle's travel direction and the maximum slope direction, the angle held by the steering organs, the presence of frontal/rear/lateral mounted implements, the additional mass of eventual carried loads, eventual suspended or movable loads (movable masses).

\section{CRITICAL ANALYSIS OF ACTUAL STABILITY TESTS}

The tests that now are normally proposed by norms to characterize the stability of an agricultural machine are substantially of two types, both simple and effective but only static: (1) static tests of lateral overturning of a vehicle in a straight-ahead travelling configuration; (2) static tests of lateral overturning of a vehicle in a specific steering configuration (this is a complementary test for articulated farm tractors only).

In both the types of tests, a vehicle, equipped with its own tires, with all the tanks completely filled with their operating liquids and loaded on its seat with some weights to simulate the presence of the driver, is placed on a inclinable flat platform and the angle of the platform necessary to have a side overturning of the vehicle is identified; it is the angle corresponding to the loss of contact with the soil of the first wheel of the vehicle. As already specified, articulated tractors are tested also according to the second type of test, to have a more complete characterization. This test is inspired by norms forecasted for telehandlers, pallet stackers, double stackers and order-picking trucks [13, 14], having also an articulated frame with a central steering joint: the tractor is placed on a tiltable support in a specific turning configuration, geometrically determined by the passage of an internal wheel's axis of the part downward (the rear part of the tractor) through the centre of external wheel of the part upward (Fig. 2). The platform is angled until the first wheel loses the contact with the supporting plane, as performed in the usual tests with a vehicle in the straight-ahead travelling configuration. Indeed, turning manoeuvres for articulated vehicles are particularly critical for the safety and deserve to be further investigated: the two halves of the frame are angled and the supporting polygon could be considerably modified [15-18]. These two tests have evident limits: vehicles are tested under static conditions at the side-overturning only, with their steering members (wheels, central joint) in a configuration corresponding to a straight-path travelling or in a specific turning angle, i.e. two very particular situations occurring during a machine's operating day. The investigation of a single steering configuration among all possible ones spanning from 0 to the maximum steering angle, is a proof of an effort done by the legislator and the experimenters to gain greater knowledge of the vehicle's behaviour on slopes (it is an advancement with respect to the straight-configuration lateral stability tests), but it is not enough. Indeed, the inquired turning configuration is too much specific because of the angulation (geometrically fixed by tractor's dimensions) and the relative position of the two tractor's halves (with the front part upward). The equation reported hereinafter shows the heavy-dependence of the inquired steering configuration on the support polygon's dimensions (for symbols, see Fig. 2).

$$
\varepsilon_{3}=\varepsilon_{1}+\varepsilon_{2}=\arcsin \frac{a}{\sqrt{a^{2}+b^{2}}}+\arcsin \frac{d}{\sqrt{c^{2}+d^{2}}} \stackrel{\substack{d=a \\ c=b}}{=} 2 \cdot \arcsin \frac{a}{\sqrt{a^{2}+b^{2}}}
$$



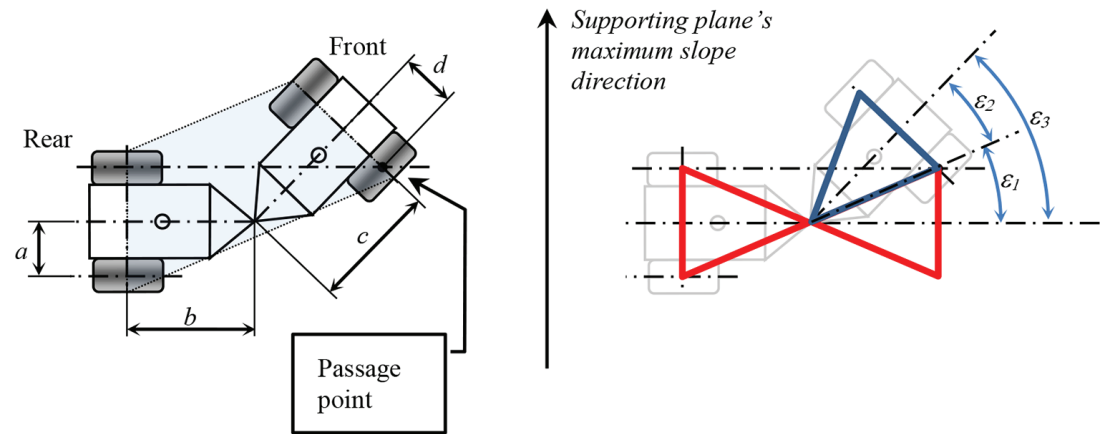

Figure 2: Specific static stability test configuration actually forecasted for articulated farm tractors on a tilting plane (seen from above), i.e. turning configuration with one of the internal wheel's axis passing through the centre of the external wheel of the part upward [13,14]; (left) the support polygon, highly distorted, in the specified turning configuration; in the same picture its characteristic dimensions are also evidenced; (right) the triangles joining the wheels' centres and the characteristic angles are visible.

This evidences another problem: although the indicated geometric configuration can be easily reproduced in its setup, it gives results that are difficultly comparable between different vehicles (similarly to what would happen if referring to the maximum steering configuration, different from a machine to another one).

Moreover, the above-described tests of lateral rollover, even if repeated on both vehicle's sides, do not allow at all localizing the COG of the vehicle neither in term of spatial position (three Cartesian coordinates), nor in relation to the vehicle's supporting base (two Cartesian coordinates): in fact, with this test it is impossible to determine the position of the COG along the longitudinal axis of the vehicle. This is a major drawback because it prevents the prediction of the stability of a vehicle when it is in conditions dissimilar from the test, e.g.: on slopes with a different inclination and with a travelling direction differently angled with respect to the maximum-slope direction.

Finally, no norm actually forecasts dynamic tests, and this is another serious limit of them. Apart from the appearance of a centrifugal force applied on the vehicle's COG, there are also phenomena very difficult to be predicted a priori even if the COG position is known from static tests and, therefore, they should be inquired experimentally. In fact, the elasticity/compressibility of some components (tires, suspensions, cabin's supports) or the presence of masses free to move within some limits (suspended loads, liquids within vessels, inconsistent solids such as grain products in a dumper, stacked solids such as pseudo-spherical fruits) cause the machine's trim to undergo a readjustment that is responsible for an accentuation of the load-transfer phenomena related to the velocity factor.

Therefore, our first objective was to conceive new tests that can overcome the above-highlighted limitations of actual test procedures. Then, at the same time, we want to present an innovative test facility that can be used to perform these new tests in a controlled and safe environment. 


\section{DEVELOPMENT OF NEW STABILITY TESTS AND OF AN INNOVATIVE TEST FACILITY (SYSTEM DEFINITION AND CONCEPT)}

The development of a new type of test takes place through a 4-step process (Fig. 3), which starts necessarily from a schematization of reality (here: an agricultural vehicle in a condition of incipient overturning), similarly to what is normally done in the field of applied sciences (physics or engineering) when deciding to investigate a phenomenon observed in reality. Subsequently, there are further steps to be done, consisting, respectively, in the individuation and isolation of the issue of interest and in the subsequent design of a test that recreates the critical situation without disturbance factors. The purpose of the test-equipment design is therefore to create an artificial situation in which the object of the test (here: the agricultural vehicle) is solicited in a controlled manner and, therefore, generates an intelligible and reproducible response.

On the basis of the 4-step process illustrated here, it is therefore necessary to start from the three previously-described overturning conditions, which actually schematize the reality by referring it to only three scenarios; each of them will be considered singularly and suitable tests to reproduce them will be conceived:

1. Excessive ground-gradient; the most suitable test equipment acts on the inclination of the support plane for the machine, and it leads a motionless machine to rollover at different values of the above-displayed variables (first of all: the angle formed by the vehicle's travelling direction with respect to the maximum slope direction); the required equipment is therefore a tiltable plane (all actual test facilities are of this type); the real advancement is letting the experimenters know which is the part of the vehicle's weight on each wheel and, hence, the position of the COG in relation to the vehicle's supporting base (two Cartesian coordinates referred to a frame of reference centred on the vehicle); this could be done only by integrating in the support plane a weighting system per wheel, possibly in a cross-shape configuration to better adapt to a wide range of wheelbases and track widths; if the integrated weighting system can also rotate along a vertical axis, it is possible to change easily the angle between the vehicle's longitudinal plane and the maximum slope direction; the required equipment is therefore a tiltable turntable;

2. Critical surface harshness; since reality has a very high variability of situations at this regard, the most critical one deserves to be investigated: it corresponds to the presence of a slope change at half of a turning trajectory (a typical situation that a tractor experiments when exiting from an inter-row), therefore with a loss of contact of the most external wheel; in this case, the most suitable test equipment uses a support plane divided into two halves and acts on their inclination: by changing the inclination of the two half planes independently, it is possible to create a slope change and then an edge; the required equipment is therefore an angleable plane;

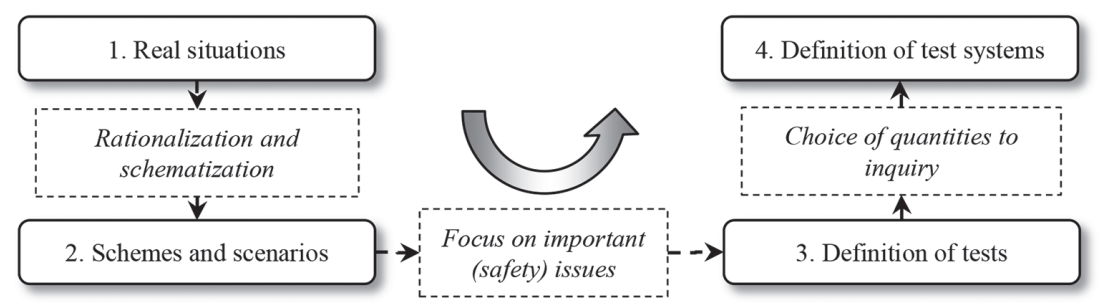

Figure 3: 4-step process of test systems' definition starting from real situations (in this case: the real working situations of a farm tractor). 
3. Excessive turning speed; the most suitable test equipment is a tiltable plane that is wide enough to let a vehicle (eventually equipped also with a trailer) travel along complete circular trajectories with increasing speeds and at different inclinations of the surface.

Summarizing, a single test-facility able to inquiry all the three critical (static and/or dynamic) overturning situations on real-scale agricultural machines (Fig. 4) conceptually integrates therefore the following test-subsystems: a tiltable plane, a tiltable turntable, an angleable plane.

The above-listed subsystems have some common points, already deducible from their designation, so the final design of the test-facility proposed here has less than three distinct test-subsystems. Indeed:

- the tiltable plane and the angleable plane are both planes that should be wide enough to let a vehicle travel on them, hence the correspondent test subsystem is a wide tiltable and angleable plane (about $15 \times 15 \mathrm{~m}$, with two degrees of freedom), having the possibility to angle itself at half of its extension, thus creating an edge useful for simulating the abrupt slope-changes that a machine can meet going out of rows in the field (Figs. 5 and 6);

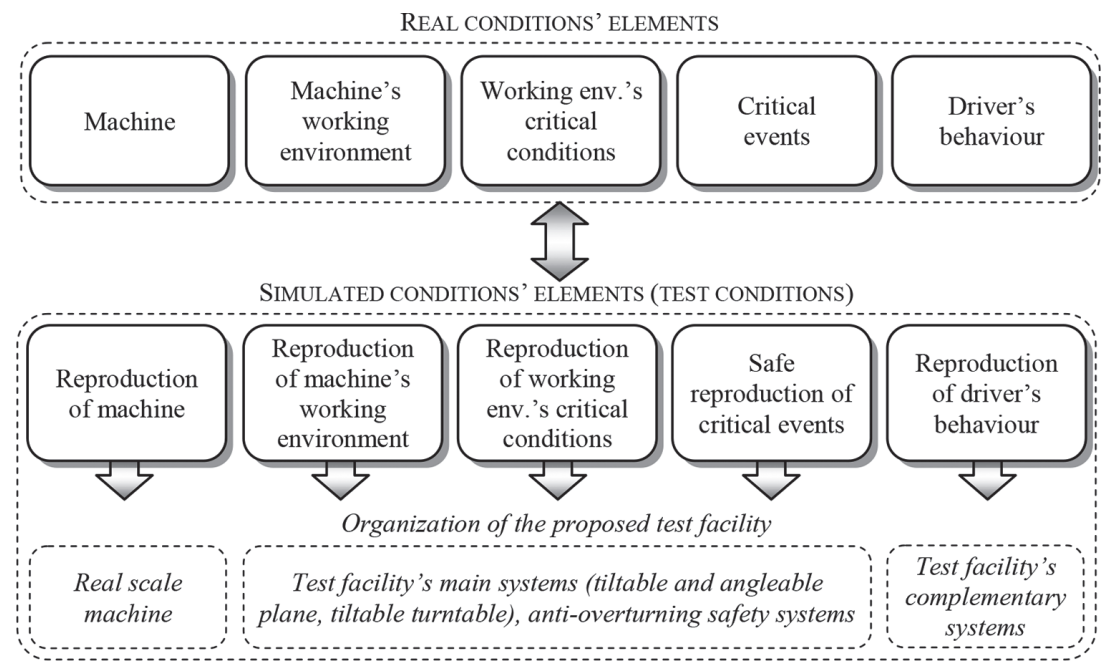

Figure 4: Main elements to be considered when setting up an experiment / an experimental layout (simulated conditions) inquiring a real situation; the picture shows also how the proposed test facility (boxes with a dotted outline) is organized in relation to the test conditions.

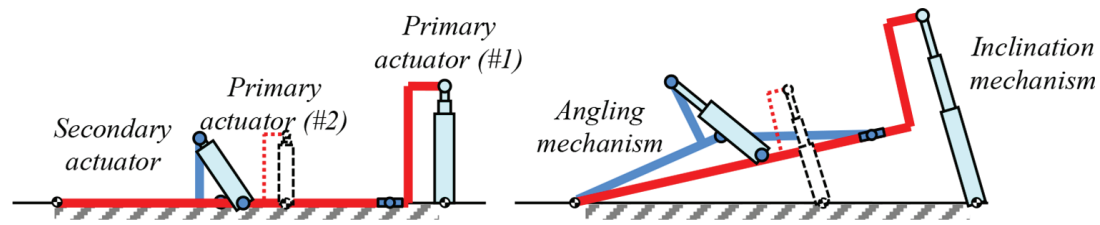

Figure 5: Schematic side views of the platform in a rest configuration (left) or tilted and angled (right). The tiltable frame is red, the secondary structure, necessary to generate the angle, is blue. If necessary, the final project can have another primary actuator (\#2) at half of the support frame, synchronized to the primary actuator (\#1). 

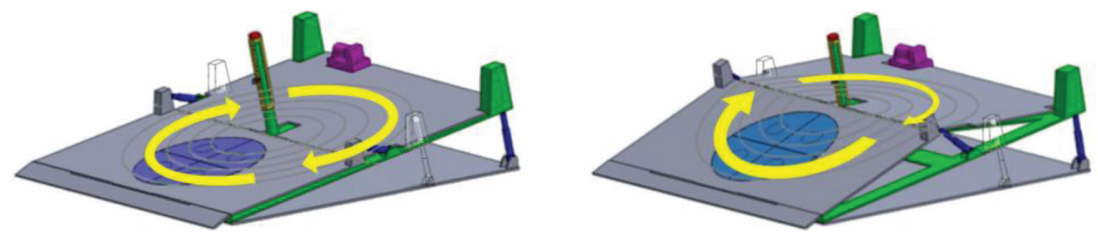

Figure 6: Axonometric views of the platform, inclined only (left) or even angled (right). In the same pictures the turntable, integrated within the lower half-platform, is evidenced with a blue colour.

- the tiltable plane and the tiltable turntable have both the capability to be inclined with respect to the supporting plane of the whole facility ('tiltable'); considering also that the tiltable turntable has a size comparable with a the size of an agricultural machine and that the tiltable plane is much wider (it allows a vehicle travelling on it along complete circular trajectories), the tiltable turntable is inserted in the tiltable plane in a way that it does not give any trouble when a vehicle travels across/on it during other tests (fully integration with lined-up surfaces; Fig. 6).

The here-proposed facility, named 'tiltable platform', is an installation having a certain complexity and it consists of several systems that can be subdivided into:

- Safety-testing systems, i.e. systems that are directly used for performing the static and dynamic stability tests discussed above (therefore, these systems have an indirect focus on safety as they are used to investigate the security performances of the tested vehicles, with the aim of increasing the knowledge concerning the tested machines, not to raise directly the safety of the object of the tests); namely, these systems are the turntable and the tiltable and angleable plane;

- Safety systems properly-said, i.e. systems that are used during the execution of the above-referred static and dynamic stability tests to ensure the safety of the whole area, in particular to ensure that no danger occurs to the involved people, to the facility, to the shed housing the facility and to the vehicle under test (therefore, these systems have a direct focus on safety, especially on the global safety of the test); among these system the most important one is the system with rotating-translating pincer-cage and carousellike arm.

\section{SYSTEM FOR THE EXECUTION OF STATIC STABILITY TESTS}

The tilting turntable is the system of the test-facility that is specifically thought for the execution of static stability tests (Fig. 7). It has the following characteristics: (1) it has a circular shape and it is divided into quadrants; each quadrant is capable of measuring the weight sustained by it due to the presence of a motionless vehicle positioned on the turntable with a wheel on each quadrant; (2) the turntable is installed on a tilting structure, and therefore it is able to simulate different gradients of the ground on which the vehicle is placed (Fig. 7; angle $a)$; (3) the turntable can rotate around an axis perpendicular to the surface supporting the vehicle, thus allowing to vary the angular position of the vehicle's longitudinal axis with respect to the maximum-slope direction of the tiltable structure (Fig. 7; angle $\varphi$ ).

When the platform is perfectly horizontal, thanks to the weights measured by each quadrant, this equipment will allow precisely locating the COG of a vehicle in relation to the 


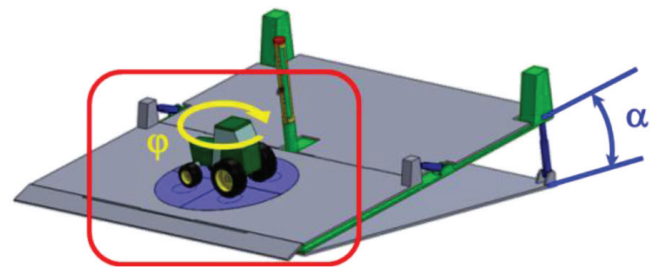

Figure 7: Static tests use only the turntable inserted in the lower half-platform; the same picture evidences the angles which characterize the spatial position of the machine under test ( $\alpha$ : global inclination of the support plane; $\varphi$ : angle between the vehicle's longitudinal plane and the maximum slope direction).

geometric centre of the supporting polygon, i.e. it is possible to have the two Cartesian coordinates of the COG, $a$ and $b$ (Fig. 8, left; WB wheelbase; FT front track; RT rear track; $\mathrm{W}_{\mathrm{FR}}$ weight at front right wheel; $\mathrm{W}_{\mathrm{FL}}$ weight at front left wheel; $\mathrm{W}_{\mathrm{RR}}$ weight at rear right wheel; $\mathrm{W}_{\mathrm{RL}}$ weight at rear left wheel; $\mathrm{W}_{\mathrm{TOT}}$ total weight of the machine):

$$
\left\{\begin{array}{l}
a=\frac{1}{2} \cdot \frac{W B \cdot\left(W_{F R}+W_{F L}-W_{R R}-W_{R L}\right)}{W_{t o t}} \\
b=\frac{1}{2} \cdot \frac{F T \cdot\left(W_{F L}-W_{F R}\right)+R T \cdot\left(W_{R L}-W_{R R}\right)}{W_{t o t}}
\end{array}\right.
$$

Subsequently, by inclining the platform with the agricultural machine's longitudinal axis parallel or perpendicular to the maximum slope direction, it is also possible to quantify the weight transfer and then to calculate the height of the COG with respect to the support plane (Fig. 8, right).

Thanks to the capability of the turntable to rotate around its vertical axis with the machine directly placed above it, it is possible to simulate a lot of working conditions of agricultural machines on slopes, corresponding to $0 \leq \varphi \leq 360^{\circ}$ and $0 \leq a \leq a_{\max }$.
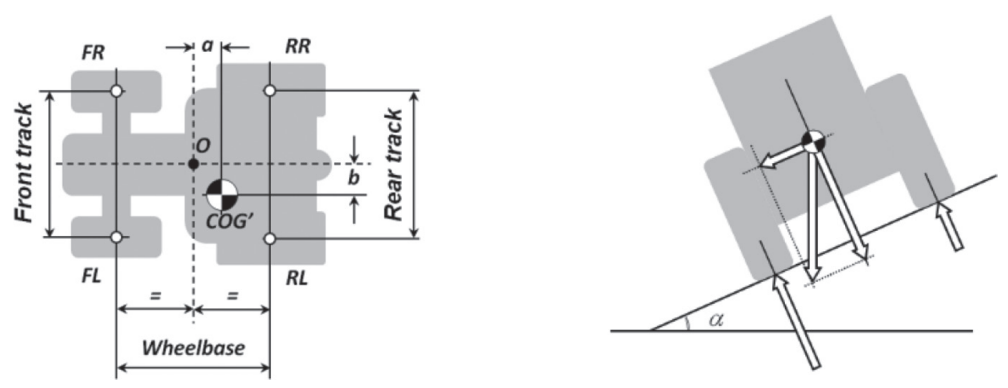

Figure 8: (Left) localization of the COG projection on the support plane (point COG') thanks to the weights on each quadrant with the tiltable plane in the horizontal position, system viewed from above; (right) localization of the COG height thanks to the quantification of the load transfer ( $\alpha$ : global inclination of the support plane). 


\section{SYSTEM FOR THE EXECUTION OF DYNAMIC STABILITY TESTS}

The whole tiltable and angleable plane of the platform, having wide dimensions (about $15 \times 15 \mathrm{~m}$ ), is used for performing dynamic stability tests (Fig. 9). These tests are organized as follows: after setting up an inclination of the plane (or of the two half-planes; Fig. 9, right), an agricultural vehicle travels on it along complete circular paths, with several radii and at speeds comparable with the speeds used by that machine on the fields.

Considering the tests on a flat surface (Fig. 9, left), when a machine travels along a complete circumference, its longitudinal axis is lined up with the tangent at each point of the circular trajectory and, in particular, assumes all the possible angles between $-180^{\circ}$ and $+180^{\circ}$ with respect to the maximum slope direction of the support surface. Therefore, with a single closed path it is possible to test the same machine in correspondence with different angles $\varphi$. If an overturning occurs, it is associated with the plane slope $a$ (responsible for the gravity force decomposition), with the angular position $\varphi$ (responsible for the rotation of the support polygon on the support plane with reference to the maximum slope direction) but also with the turning radius $r$ and the speed $v$ (responsible for the centrifugal force intensity and for the weight transfer; Fig. 10).

Dynamic tests on an angled surface have the upper half-platform horizontal (Fig. 9, right) and the lower half-platform inclined. The artificial edge divides each circular trajectory making use of the whole extension of the platform and simulates the sharp slope-changes that a machine can meet in a field, for example going out of the rows (Fig. 11). This is a situation worthy to be inquired because of the impossibility for some vehicles to fully comply with the inclined plane, leading to the loss of contact of a wheel (typically the most external wheel, in position C/C' of Fig. 11, left).
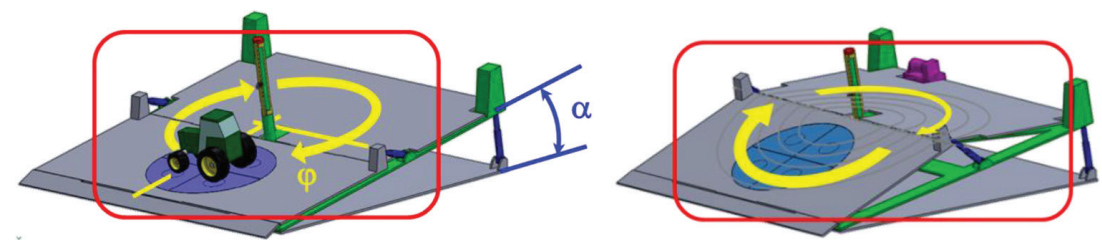

Figure 9: Dynamic tests on a flat (left) or on an angled (right) surface use the whole plane of the tiltable platform; in the picture on the left the angles which characterize the spatial position of the machine under test are evidenced. The reported trajectories (dark grey in the picture on the right) will be travelled as indicated by the yellow arrows during dynamic tests (radii: $2.5,3.5,4.5,5.5,6.5 \mathrm{~m}$ ).
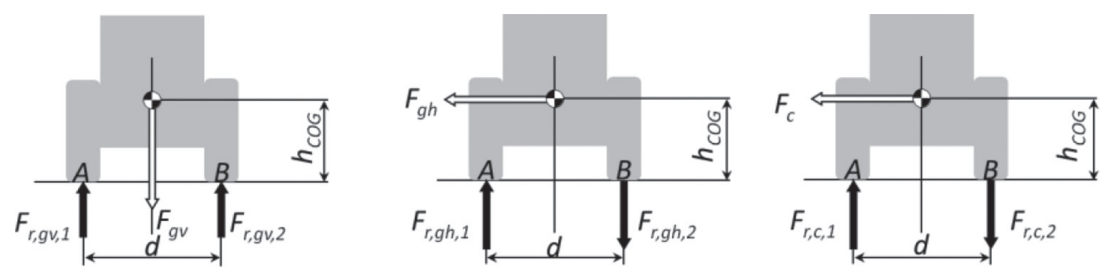

Figure 10: Effect of the gravity force decomposition (left/centre) and of the centrifugal force (right) on the reactional forces (not in scale) developed by the support in correspondence with the wheels of the same vehicle (seen in a plane transversal to the vehicle's longitudinal axis); the slope of the plane increases toward $\mathrm{B}$, the turning manoeuvre has the curvature centre at the right side of the pictures. 


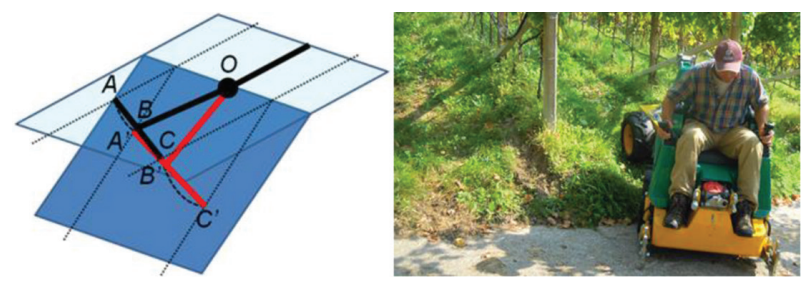

Figure 11: (Left) schematization of an articulated vehicle astride the edge (O: articulation joint; thick black/red lines: vehicle frame in the horizontal/inclined plane); the front half/axle $(\mathrm{ABC})$ is subjected to a double inclination (on the horizontal plane, to turn, and also due to the slope change); (right) example of a real vehicle (a small articulated tractor) undergoing the described situation [18].

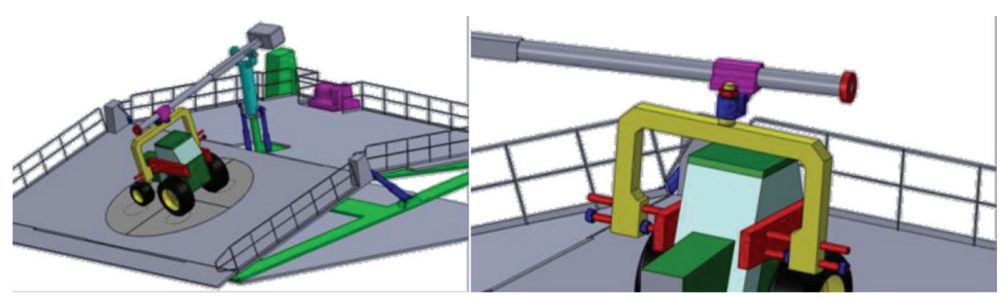

Figure 12: System to prevent the free overturning of the vehicle under test.

\section{ANTI-OVERTURNING SYSTEM FOR THE TRACTOR UNDER TEST}

The execution of real-scale dynamic tests with machines having considerable masses (according to the platform project: up to $5000 \mathrm{~kg}$ ) raises very important issues regarding the general safety. The tests to be conducted on this equipment aim at verifying the machines' stability in several conditions, so they can lead the vehicle to the overturning. However, a free, unstopped overturning must be avoided at all to prevent serious damages to people and things near the facility, due to the dangerous condition of having a relevant mass in a not-controlled motion within an enclosed area. Therefore, a retaining system for the machine in test has been studied together with the other above-explained (test) systems.

The spatial trajectory that a vehicle can travel during a test on this innovative platform is perfectly circular only when the support-plan is not angled. Starting from this consideration and studying the possible motions and trajectories of a tested vehicles, we therefore propose a system that can provide up to 6 degrees-of-freedom and block selectively some of them in case of danger. Looking at its technical characteristics, it can be named to as: system with rotating-translating pincer-cage and carousel-like arm (Fig. 12).

\section{CONCLUSIONS}

This study presents a test-facility thought to perform a series of new stability tests on agricultural vehicles, called the 'tiltable platform'. It has been conceived starting from a deep analysis of the stability issues, of the rollover causes for agricultural machines and of actual stability tests, evidencing their limits. A special focus has been devoted to the characterization tests of articulated vehicles' stability, due to the high distortion of their support polygon when turning. Using a 4-step process, each of the three scenarios that actually schematize the real 
overturning conditions has been considered singularly, then new tests and test-systems, suitable to reproduce these tests, have been conceived, originating the new-concept test-facility presented here. It includes some innovative solutions for performing static tests different from actual ones (the tilting turntable), and also for performing dynamic tests (the tiltable and anglable plane), thus allowing a complete/global evaluation of the agricultural vehicles (e.g., including the position of the COG, the measurement of the load distribution and load transfer).

\section{REFERENCES}

[1] Mazzetto, F., Didactic material from the lessons of agricultural mechanics and mechanization, 2017.

[2] Lee, T.Y., Gerberich, S.G., Gibson, R.W., Carr, W.P., Shutske, J. \& Renier, C.M., A population-based study of tractor-related injuries: Regional Rural Injury Study-I (RRIS-I). Journal of Occupational \& Environmental Medicine, 38, pp. 782-793, 1996. https://doi.org/10.1097/00043764-199608000-00014

[3] Myers, M.L., Tractor risk abatement and control as a coherent strategy. Journal of Agricultural Safety and Health, 8, pp. 185-198, 2002. https://doi.org/10.13031/2013.8431

[4] Erlich, S.M., Driscoll, T.R., Harrison, J.E., Frommer, M.S. \& Leigh, J., Work-related agricultural fatalities in Australia, 1982-1984. Scandinavian Journal of Work, Environment \& Health, 19, pp. 162-167, 1993. https://doi.org/10.5271/sjweh.1486

[5] Bernik, R. \& Jerončič, R., The research of the number of accidents with the agriculture and forestry tractors in the Europe and the main reasons for those accidents. Strojniški vestnik: Journal of Mechanical Engineering, 54, pp. 557-564, 2008.

[6] HSE - Health and Safety Executive. Health and safety in agriculture, forestry and fishing in Great Britain, 2014/15, 2015.

[7] INAIL. Report annuale sugli infortuni mortali e con feriti gravi verificatisi nel 2014 nel settore agricolo e forestale. 2015.

[8] Chisholm, C.J., The effect of parameter variation on tractor overturning and impact behaviour. Journal of Agricultural Engineering Research, 24, pp. 417-440, 1979. https://doi.org/10.1016/0021-8634(79)90081-7

[9] Chisholm, C.J., A mathematical model of tractor overturning and impact behaviour. Journal of Agricultural Engineering Research, 24, pp. 375-394, 1979. https://doi.org/10.1016/0021-8634(79)90079-9

[10] Coombes, G.B., Slope stability of Tractors. Farm Machine Design Engineering (Design Engineering Publications), p. 18-33, 1968.

[11] Guzzomi, A.L., A revised kineto-static model for Phase I tractor rollover. Biosystems Engineering, 113, pp. 65-75, 2012.

https://doi.org/10.1016/j.biosystemseng.2012.06.007

[12] Myers, M.L., Continuous overturn control of compactors/rollers by rollover protective structures. International Journal of Vehicle Safety, 3, p. 45, 2008. https://doi.org/10.1504/IJVS.2008.020079

[13] UNI ISO. UNI ISO 22915-4:2012 Carrelli industriali - Verifica della stabilità - Parte 4, 2012.

[14] UNI EN. UNI EN 1459-3:2015 Carrelli elevatori fuoristrada - Requisiti di sicurezza e verifica - Parte 3, 2015. 
[15] Bietresato, M., Carabin, G., Vidoni, R., Mazzetto, F. \& Gasparetto, A., A parametric approach for evaluating the stability of agricultural tractors using implements during side-slope activities. Contemporary Engineering Sciences, 8, pp. 1289-1309, 2015. https://doi.org/10.12988/ces.2015.56185

[16] Vidoni, R., Bietresato, M., Gasparetto, A. \& Mazzetto, F., Evaluation and stability comparison of different vehicle configurations for robotic agricultural operations on side-slopes. Biosystems Engineering, 129, pp. 197-211, 2015. https://doi.org/10.1016/j.biosystemseng.2014.10.003

[17] Mazzetto, F., Bietresato, M. \& Vidoni, R., Development of a dynamic stability simulator for articulated and conventional tractors useful for real-time safety devices. Applied Mechanics and Materials, 394, pp. 546-553, 2013. https://doi.org/10.4028/www.scientific.net/AMM.394.546

[18] Mazzetto, F., Bietresato, M., Gasparetto, A. \& Vidoni, R., Simulated stability tests of a small articulated tractor designed for extreme-sloped vineyards. Journal of Agricultural Engineering, XLIV, pp. 663-668, 2013.

https://doi.org/10.4081/jae.2013.(s1):e133 